\title{
BMJ Open Computerised decision to reduce inappropriate medication in the elderly: a systematic review with meta- analysis protocol
}

\author{
Luís Monteiro, ${ }^{1,2}$ Tiago Maricoto, ${ }^{1,3}$ Isabel S Solha, ${ }^{4}$ Matilde Monteiro-Soares, ${ }^{5,6}$ \\ Carlos Martins ${ }^{5,6}$
}

To cite: Monteiro L, Maricoto T, Solha IS, et al. Computerised decision to reduce inappropriate medication in the elderly: a systematic review with metaanalysis protocol. BMJ Open 2018;8:e018988. doi:10.1136/ bmjopen-2017-018988

- Prepublication history for this paper is available online. To view these files, please visit the journal online (http://dx.doi org/10.1136/bmjopen-2017018988).

Received 7 August 2017 Revised 29 November 2017 Accepted 13 December 2017

Check for updates

${ }^{1}$ Faculdade de Ciências da Saúde, Universidade da Beira Interior, Covilhã, Portugal ${ }^{2}$ USF Esgueira +, Aveiro, Portugal

${ }^{3}$ USF Aveiro/Aradas, Aveiro, Portugal

${ }^{4}$ USF Terras de Souza, Paredes, Portugal

${ }^{5}$ Departamento de Medicina da Comunidade, Informação e Decisão em Saúde, Oporto University Faculty of Medicine, Oporto, Portugal

${ }^{6}$ Center for Health Technology and Services Research (CINTESIS), Oporto University Faculty of Medicine, Oporto, Portugal

Correspondence to

Dr Luís Monteiro;

luismonteiro.net@gmail.com

\section{ABSTRACT}

Introduction Life expectancy continues to increase in developed countries. Elderly people are more likely to consume more medications and become vulnerable to age-related changes in drugs' pharmacokinetics and pharmacodynamics. Recent studies have identified opportunities and barriers for deprescribing potentially inappropriate medications. It has already been demonstrated that computerised decision support systems can reduce physician orders for unnecessary tests. We will systematically review the available literature to understand if computerised decision support is effective in reducing the use of potentially inappropriate medications, thus having an impact on health outcomes.

Methods and analysis A systematic review will be conducted using MEDLINE, CENTRAL, EMBASE and Web of Science databases, as well as the grey literature assessing the effectiveness of computer decision support interventions in deprescribing inappropriate medication, with an impact on health outcomes in the elderly. The search will be performed during January and February 2018. Two reviewers will conduct articles' screening, selection and data extraction, independently and blind to each other. Eligible sources will be selected after discussing non-conformities. All extracted data from the included articles will be assessed based on studies' participants, design and setting, methodological quality, bias and any other potential sources of heterogeneity. This review will be conducted and reported in adherence to the Preferred Reporting Items for Systematic Reviews and Meta-Analyses statement of quality for reporting systematic reviews and meta-analyses.

Ethics and dissemination As a systematic review, this research is exempt from ethical approval. We intend to publish the full article in a related peer-reviewed journal and present it at international conferences.

PROSPEROregistration number CRD42017067021.

\section{INTRODUCTION}

In developed countries, ageing population is increasing. ${ }^{1}$ Caring for older adults is a challenge for healthcare providers, as they are
Strengths and limitations of this study

We aim to clarify whether new technologies, namely computerised decision systems, can help in reducing inappropriate medication in the elderly.

- This protocol was written following the recently published Preferred Reporting Items for Systematic Review and Meta-Analysis Protocols guidelines.

- We will conduct a comprehensive systematic review on this clinical topic using, if possible, meta-analytic methods.

- Studies with high heterogeneity and varying quality may limit the quality of evidence for this systematic review.

more likely to have multimorbidities ${ }^{23}$ and to consume more medication. ${ }^{4}$

Polypharmacy, defined as 'the use of multiple drugs administered to the same patient, most commonly seen in elderly patients, ${ }^{56}$ although frequent has a negative impact on senior health. ${ }^{78}$ There is an increased risk of drug interactions and prescriptions of potentially inappropriate medications, ${ }^{4}$ changes in pharmacokinetics and pharmacodynamics and limited generalisation of clinical research results due to common exclusion of subjects aged more than 65 years old. ${ }^{9}$ So, prescribing medication for elderly patients should be evidence based and particularly cautious.

In several cases it is urgent to deprescribe, this is to begin 'the process of withdrawal of inappropriate medication, supervised by a health care professional with the goal of managing polypharmacy and improving outcomes. $^{10}$

Inappropriate medication prescription, meaning "the practice of administering medications in a manner that poses more risk than benefit, particularly where safer alternatives exist, ${ }^{511}$ can be reduced by several 
interventions. ${ }^{12}$ However, they are not widely known and therefore used. In one hand, general practitioners report interest in learning and using more mobile technologies to assist in clinical care ${ }^{13}$; on the other hand, they refer an insufficient emphasis on geriatric pharmacotherapy training. ${ }^{14}$

It has already been shown that computerised decision support systems can reduce physicians' orders of unnecessary tests. ${ }^{15}$ This systematic review aims to determine if computerised decision support is effective in reducing potentially inappropriate medication prescription in the elder population.

Other studies have addressed strategies to improve care of elderly in what concerns inappropriate medication prescription. ${ }^{12} 16$ In 2013, one synthesis study identified eight randomised controlled trials (RCT), two cluster RCTs and two controlled before-and-after studies. ${ }^{9}$ In 2015, another study included 12 RCTs. ${ }^{13}$ Both studies reported high heterogeneity on the included studies. However, these studies have not focused on computerised decision support systems. In addition, we consider that since the last study search, more adequate studies have been published and that, for the first time, a meta-analysis will be possible to conduct.

\section{METHODS AND ANALYSIS \\ Eligibility criteria}

In this systematic review, we will select (1) interventional studies, such as RCTs, non-randomised controlled studies and quasirandomised controlled studies; (2) those that include participants aged 65 years or more, to whom one or more regular medications were prescribed, and (3) assess the impact of computerised decision support systems in withdrawal of potentially inappropriate medication prescription. On the other hand, studies including only moribund, terminal or palliative participants will be excluded. Studies published or in press will be included independent of the language, year of publication and setting in which it was conducted (hospitals, nursing centres, communities, and so on). Potentially inappropriate medications will be defined using the Beers criteria $^{17}$ and STOPP/START criteria. ${ }^{18}$

\section{Information sources}

Our sources of information will include electronic databases (namely MEDLINE, CENTRAL, EMBASE, Web of Science), trial registries, different types of grey literature and contact with specialists in the field. If further data are needed, authors of the selected articles will be contacted. The search will be performed in January and February 2018. The search will have no language restrictions. In those cases that none of the research team members are able to translate the included study, we will first contact the authors to ascertain if the main data are available in other languages and seek to translate whenever necessary. A second search using all identified keywords and proprietary names of computerised decision support systems will then be undertaken across all included databases.

\section{Search strategy}

Our initial search syntax in CENTRAL will be: (1) MeSH descriptor: [Medical Informatics Applications] explode all trees; (2) Computer decision support; (3) MeSH descriptor: [Deprescriptions] explode all trees; (4) MeSH descriptor: [Inappropriate Prescribing] explode all trees; (5) no. 1 or 2; (6) no. 3 or 4; (7) no. 5 and no. 6.

For PubMed, the query will be "(Medical Informatics Applications [MeSH Terms] OR (medical AND informatics AND applications)) AND ((Deprescriptions [Mesh Terms] OR deprescription OR deprescribing OR Inappropriate Prescribing [Mesh Terms] OR (inappropriate AND prescribing*) OR (inappropriate AND prescription*) OR (over* AND prescribing*)) OR medication errors [MeSH Terms] OR (error* AND medication) OR (drug AND use AND error*) AND (decision support systems, clinical [MeSH Terms] OR 'clinical decision support systems' OR (clinical AND decision AND support*) OR decision making, computer-assisted [MeSH Terms] OR (computer AND assisted AND decision AND making) OR (medical AND computer AND assisted AND decision AND making) OR medical order entry systems [MeSH Terms] OR (medical AND order entry systems) OR (medications AND alert AND systems) OR 'computorized physician order entry systems' OR 'computorized provider order entry systems' OR 'computorized physician order entry' OR 'computorized provider order entry')."

For Web of Science the query will be "TS=('Medical Informatics Applications' OR (medical AND informatics AND applications $))$ AND TS $=(($ Deprescriptions OR deprescription OR deprescribing OR 'Inappropriate Prescribing' OR (inappropriate AND prescribing*) OR (inappropriate AND prescription*) OR (over* AND prescribing*)) OR 'medication errors' OR (error* AND medication) OR (drug AND use AND error*) AND TS $=$ ('clinical decision support systems' OR (clinical AND decision AND support*) OR decision making, computer-assisted [MeSH Terms] OR (computer AND assisted AND decision AND making) OR (medical AND computer AND assisted AND decision AND making) OR 'medical order entry systems' OR (medical AND order entry systems) OR (medications AND alert AND systems) OR 'computorized physician order entry systems' OR 'computorized provider order entry systems' OR 'computorized physician order entry' OR 'computorized provider order entry').”

\section{Study selection process}

The selection process procedure will be made by two reviewers following several steps.

First, they will independently review the title and abstract of each reference. Each one will be categorised into either relevant, unsure or irrelevant. If a reference 
is considered irrelevant by the two authors it will be eliminated.

In the next phase, the two authors will review the full text of the remaining references and each one will independently select which articles should be included.

The two authors will compare their selected articles and discuss any disagreement in each phase.

If the two reviewers cannot reach an agreement all the authors of the paper will make the final decision.

\section{Data extraction and management}

Once the articles to be included are selected, data will be extracted and entered into data sheets independently by two reviewers. These two sheets, including their differences, will be checked by a third reviewer.

The following information will be extracted from each article: (1) study characteristics, intervention type; type of study; country, setting, follow-up duration; (2) participants' number and age; and (3) clinical outcomes. The primary outcome to be considered is the effect of intervention on withdrawal of potentially inappropriate medications (discontinuation rate). The authors will give priority to the following outcomes, by order of importance: mortality, hospitalisation, any reported adverse drug withdrawal effects and quality of life measurements.

Any potential difference among reviewers will be discussed with the team, and if not resolved, the manuscript authors will be contacted. Also, if required data are missing from the article or are incomplete or unclear, inquiries will similarly be sent to the authors.

\section{Risk of bias}

Two reviewers will assess, independently and blinded to each other, the risk of bias by applying the Cochrane Collaboration Risk of Bias tool to all the included studies. ${ }^{19}$

\section{Data synthesis}

The final report will present the available data of the computer decision to support in reducing inappropriate medication prescription in older adults.

Each outcome will be combined and calculated using the statistical software RevMan V.5.1, ${ }^{20}$ according to statistical guidelines referenced in the current version of the Cochrane Handbook for Systematic Reviews of Interventions. $^{21}$

If we are able to include a group of studies that are sufficiently comparable and reliable we will conduct a meta-analysis. We consider that we should use a random effects model taking in consideration the previous systematic reviews' results. We expect to encounter a sufficient number of studies, reporting a sufficient number of events, but that are not completely comparable (concerning the intervention, context and population).

If heterogeneity is severe ( $\mathrm{I}^{2}$ superior to $\left.40 \%-50 \%\right)$ and studies' results are strongly biased, we will not perform a meta-analysis; thus, a narrative, qualitative summary will be done instead.
Effect sizes and 95\% CI will be expressed as ORs. When a study reports zero event in both arms, we will consider using zero-cell correction methods.

Subgroup analyses will be used to explore possible sources of heterogeneity based on the following: setting, type of software, medication and participants' clinical characteristics.

Regarding subgroups, we assume it will be relevant to include subgroups regarding the tool used by software to identify targets: STOPP/START criteria subgroup and the Beers criteria. We will also conduct metaregression to evaluate whether the covariates have significant influence on heterogeneity.

Forest plots will be produced when three or more studies are included in a meta-analysis. Data in tables will be presented by therapeutic class based on the Anatomical Therapeutic Classification codes.

Studies rated as having a high risk of bias will be included in the narrative synthesis but not on our meta-analysis and discussed in detail.

A systematic narrative synthesis will be provided in the text and tables to summarise and explain the characteristics and findings of the studies; it will explore the relationship within and between studies, in line with guidance from the Centre for Reviews and Dissemination.

To determine whether publication bias is present, we will include funnel plot and statistical tests in the assessment, namely Begg's test and Egger's test.

We will also ascertain if each RCT had its protocol published before recruitment of patients was initiated.

The quality of evidence for all outcomes will be judged with the Grading of Recommendations Assessment, and the Development and Evaluation working group methodology. ${ }^{22}$

The final paper will be prepared following the Preferred Reporting Items for Systematic Reviews and Meta-Analyses guidelines. ${ }^{23} 24$

\section{ETHICS AND DISSEMINATION}

As a systematic review, this research is exempt from ethical approval. We intend to publish the full article in a related peer-reviewed journal and present it in international conferences.

\section{DISCUSSION}

Although electronic health records are common in clinical practice, there is a lack of evidence of computer decision support systems regarding health outcomes. Deprescribing potentially inappropriate medication in the elderly is particularly difficult, although computer support may be an important tool. This systematic review will help identify the success of computerised decision support to reduce inappropriate medication prescription. Therefore, this review will be relevant for patients, health professionals and policymakers. One potential limitation of this study will be if we find a limited number of studies 
with considerable differences regarding their characteristics and methodology. This may impair our conclusions and impede meta-analysis. In addition, depending on the data available and obtained results we may not be able to define which is the best decision support available.

Contributors LM had the original idea for the systematic review. LM, TM and ISS wrote the protocol and reviewed the search strategy. LM, TM, ISS, MMS and CM reviewed the protocol.

Funding This article was supported by FEDER through POCI-01-0145FEDER-007746, funded by the Programa Operacional Competitividade e Internacionalização-COMPETE2020 and by National Funds through FCTFundação para a Ciência e a Tecnologia within CINTESIS, R\&D Unit (Reference UID/IC/4255/2013). MMS' work is financed by Project 'NORTE-01-0145FEDER-000016' (NanoSTIMA), financed by the North Portugal Regional Operational Programme, NORTE 2020, under the PORTUGAL 2020 Partnership Agreement and the European Regional Development Fund (ERDF).

Competing interests None declared.

Patient consent Not required.

Ethics approval This research is exempt from ethical approval.

Provenance and peer review Not commissioned; externally peer reviewed.

Open Access This is an Open Access article distributed in accordance with the Creative Commons Attribution Non Commercial (CC BY-NC 4.0) license, which permits others to distribute, remix, adapt, build upon this work non-commercially, and license their derivative works on different terms, provided the original work is properly cited and the use is non-commercial. See: http://creativecommons.org/ licenses/by-nc/4.0/

(C) Article author(s) (or their employer(s) unless otherwise stated in the text of the article) 2018. All rights reserved. No commercial use is permitted unless otherwise expressly granted.

\section{REFERENCES}

1. OECD. Elderly population (indicator). https://data.oecd.org/pop/ elderly-population.htm\#indicator-chart (accessed 26 Feb 2017).

2. Marengoni A, Angleman S, Melis R, et al. Aging with multimorbidity: a systematic review of the literature. Ageing Res Rev 2011;10:430-9.

3. Prazeres F, Santiago L. Prevalence of multimorbidity in the adult population attending primary care in Portugal: a cross-sectional study. BMJ Open 2015;5:e009287.

4. Weng MC, Tsai CF, Sheu KL, et al. The impact of number of drugs prescribed on the risk of potentially inappropriate medication among outpatient older adults with chronic diseases. QJM 2013;106:1009-15.

5. Grosjean J, Merabti T, Dahamna B, et al. Health multi-terminology portal: a semantic added-value for patient safety. Stud Health Technol Inform 2011;166:129-38.

6. HeTOP [Internet]. Rouen University Hospital. 2017 http://www.hetop. eu/hetop/?la=en\&q=\#la=en\&rr=MSH_D_019338\&q=polypharmacy (cited 17 Mar 2011 (SCHEME=ISO8601)).
7. Fried TR, O'Leary J, Towle V, et al. Health outcomes associated with polypharmacy in community-dwelling older adults: a systematic review. J Am Geriatr Soc 2014;62:2261-72.

8. Lu WH, Wen YW, Chen LK, et al. Effect of polypharmacy, potentially inappropriate medications and anticholinergic burden on clinical outcomes: a retrospective cohort study. CMAJ 2015;187:E130-7.

9. Van Spall HG, Toren A, Kiss A, et al. Eligibility criteria of randomized controlled trials published in high-impact general medical journals: a systematic sampling review. JAMA 2007;297:1233-40.

10. Reeve E, Gnjidic D, Long J, et al. A systematic review of the emerging definition of 'deprescribing' with network analysis: implications for future research and clinical practice. $\mathrm{Br} \mathrm{J}$ Clin Pharmacol 2015;80:1254-68.

11. HeTOP. Inappropriate prescribing [Internet]. $2017 \mathrm{http}: / /$ www.hetop.eu/hetop/?la=en\&q=\&home\#la=en\&rr=MSH_D_ 057970\&q=inappropriate+prescribing (cited 17 Mar 2011 (SCHEME=ISO8601)).

12. Patterson SM, Cadogan CA, Kerse N, et al. Interventions to improve the appropriate use of polypharmacy for older people. Cochrane Database Syst Rev 2014:10.

13. survey PCISoW. PCI Survey: Family doctors want education on a smartphone. 2017 https://www.dropbox.com/s/4vy5o2kdhz7ji8z/ WONCA\%20survey\%20summary\%20ACv2.pdf?dl=0.

14. Cullinan S, Fleming A, O'Mahony D, et al. Doctors' perspectives on the barriers to appropriate prescribing in older hospitalized patients: a qualitative study. Br J Clin Pharmacol 2015;79:860-9.

15. Martins CM, da Costa Teixeira AS, de Azevedo LF, et al. The effect of a test ordering software intervention on the prescription of unnecessary laboratory tests - a randomized controlled trial. BMC Med Inform Decis Mak 2017;17:20.

16. Clyne B, Fitzgerald C, Quinlan A, et al. Interventions to address potentially inappropriate prescribing in community-dwelling older adults: a systematic review of randomized controlled trials. J Am Geriatr Soc 2016;64:1210-22.

17. By the American Geriatrics Society 2015 Beers Criteria Update Expert Panel. American Geriatrics Society 2015 updated beers criteria for potentially inappropriate medication use in older adults. $J$ Am Geriatr Soc 2015;63:2227-46.

18. O'Mahony D, O'Sullivan D, Byrne S, et al. STOPP/START criteria for potentially inappropriate prescribing in older people: version 2. Age Ageing 2015;44:213-8.

19. Higgins JP, Altman DG, Gøtzsche PC, et al. The cochrane collaboration's tool for assessing risk of bias in randomised trials. BMJ 2011;343:d5928

20. The Cochrane Collaboration. Review manager (RevMan) Version 5.3 ed. Copenhagen: The Nordic Cochrane Centre, 2014.

21. The Cochrane Collaboration. Cochrane handbook for systematic reviews of interventions version 5.1.0. London, UK: The Cochrane Collaboration, 2011

22. Alonso-Coello P, Schünemann HJ, Moberg J, et al. GRADE Evidence to Decision (EtD) frameworks: a systematic and transparent approach to making well informed healthcare choices. 1 Introduction. BMJ 2016;353:i2016.

23. Moher $D$, Shamseer $L$, Clarke $M$, et al. Preferred reporting items for systematic review and meta-analysis protocols (PRISMA-P) 2015 statement. Syst Rev 2015;4:1.

24. Shamseer L, Moher D, Clarke M, et al. Preferred reporting items for systematic review and meta-analysis protocols (PRISMA-P) 2015: elaboration and explanation. BMJ 2015;349:g7647. 